$\xi=-$ 国

\title{
Mental health analysis on digital world with meditation using EEG
}

\author{
S. Saravanan ${ }^{1 *}$, S. Govindarajan ${ }^{2}$ \\ ${ }^{1}$ Department of Computer Science and Engineering, SRM Institute of Science and Technology, Chennai, India. \\ ${ }^{2}$ Department of EDP, SRM Institute of Medical Sciences, Chennai, India. \\ E-mail:govindaraja.n@ktr.srmuniv.ac.in \\ *Corresponding author E-mail:sarnag07@gmail.com
}

\begin{abstract}
Internet, e-mail and other social networks like Myspace, Facebook, twitter, LinkedIn are the indispensable components in today's world. These social networking makes the human to addict into the digital world. Digital world has become the integral part of our society. Addiction to the digital world slowly develops the negative symptoms in the area of physical, physiological, emotional and psychological. The most affected of all is the change in Emotional behaviour of the Humans. Emotions plays an important role in our day today life. The existing research work, based on subjective self-reports shows prolonged use of Digital Media induce negative emotions for Humans. There are several techniques are used to extract the human emotions from brain such as Electroencephalography (EEG), functional Magnetic Resonance Imaging (fMRI), or Positron Emission Tomography (PET). Many of the researchers are extensively used to extract the brain waves using EEG. The negative emotions are controlled by human through meditation. In this paper, the Mind Wave device has been used to extract the EEG signal using different range of age people during they use the Digital Medias and after they perform mediation. The proposed method identify the stress level of the human while they are using social media with meditation and without meditation. It evidently proved that the meditation reduces the stress level of human.
\end{abstract}

Keywords: Digital Medias, EEG, fMRI, PET, Mind Wave.

\section{Introduction}

Social media has been creating a big impact on the teenagers these days. Social media has plenty of advantages where it allows them to connect to anyone in and around the world, share pictures and information, play online games etc. At the same time it has various drawbacks. Using social media for a long time leads to health ailments, disorders and illness.(egAlzehiemer) .Various research has proved that students using social networking sites in a regular basis has stomach pain, anxiety and depression. Therefore practicing meditation for a certain period of time will help in balancing their stress and depression level. Brain computer interface is a communication pathway between the human brain and the computer. It is distinguished into three types: 1. Invasive (Neurosurgery), 2. Semi invasive (ECoG), 3. Non invasive (EEG). In this project we have used the EEG approach. Electroencephalography (EEG) is used for recording the brain activity. The recording is done by placing the electrodes on the scalp. The neurosky device is used to sense the meditation level and the emotions of the subject.

\section{Literature survey}

Various research works has been carried out over the past few years. Roy Pea, Cliford Nass, Lyn Meheula, Marcus Rance, Aman Kumar, Holden Bamord, Matthew Nass, Aneesh Simha, Steven Yang and Micheal Zhou [8] has found the effect of technology on emotions of 8-12 year old girls in
America and Canada. Their research has been done using online questionnaire asked to parents. They concluded that those who spent more time on social media have a very bad sleep and surrounded by less people.

The work done by Hygeia Casiano, D.Jolene Kinleyr, Laurence Y.Kartz, Mariette J.Chartie, Jitender Sareen [7] focuses on examining the association between the media use and health outcomes among teenagers. Playing video games leaded to negative health issues like depression, stress and alcohol dependence.

Javier SerranoPuche [5] has done a paper that focus on emotions to the use of mobile phones. He associated the socio cultural practices similar with the use of mobile phones. Research has been done using various methods interviews and diaries written by participants.

The work of Jiequan Li and M.Oussalah [1] focuses on facial emotion recognition. It involves the use of HAAR transforms and adaptive adaboost algorithm, PCA, NMF and KNN algorithms. PCA is obtained by finding the Eigen value of a covariance matrix of data. The K-dimensional representation is given by the $y=E^{T}(x-\mu)$.

NMF is used to depict the data using non negativity constraints. This algorithm decomposes the primary matrix. Decho Surangsrirat and Apichart Intarapanich [3] has done a paper on analyzing the brainwave using the muse device. Vatsla Chauhan, M.Uma, S.Karthik, Vaibhav Nagpal [2] focuses on getting high beta waves from the subjects by extracting their EEG signals and also to generate alpha waves by playing music. 
The work of Chandana, Vijayalakshmi, Vinod Kochupillai [4] shows the relaxed state of people after meditation. This paper also proves that right part of the brain work more than the left part after meditation.

\section{Neurosky device}

The neurosky device monitors the electric impulses released in the brain. The device consists of a headset(adjustable),ear cut and a sensor arm. The device sense on measure attention, meditation and the emotions of the subjects. The device removes all the noise and the artifacts from the data hence there is no need to preprocess the data. It uses a solitary AAA battery which lasts for $8 \mathrm{hrs}$. The data is recorded using the EEGid application via Bluetooth. The raw data is in the csv format.

Attention eSense: This unsigned one byte value reports the current eSense attention meter of the user. It indicates the intensity of a users level of mental "focus" or "attention". It ranges from 0-100.Diversions, absence of center or uneasiness may bring down the attention level.

Meditation eSense This unsigned one byte value indicates the level of a users mental "calmness" or "relaxation". Its value ranges from 0-100.Diversions, meandering thoughts, tension may bring down the meditation level.

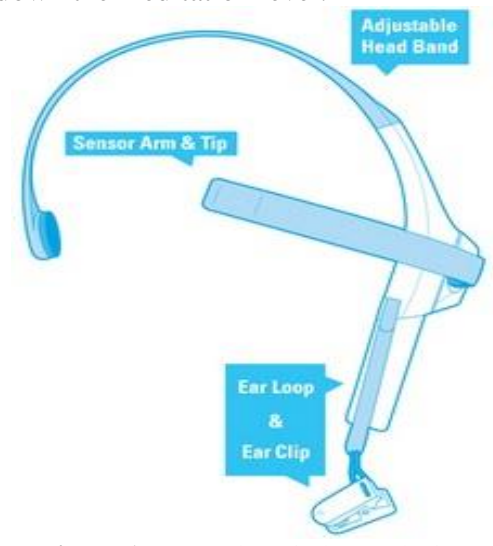

Figure 1: Neurosky mindwave device

\section{EEG brain waves}

The different brain waves obtained from the neurosky device are classified according to the frequency and amplitude. There are six types of brain waves:

1. Delta waves-These waves ranges between $0.5-4 \mathrm{hz}$. They are mainly associated with deep sleep and coma stage.[6]

2. Theta waves-These waves ranges between 47hz.Normally associated with drowsiness and frustration.[6]

3. Alpha waves-They lie within a range of $8-13 \mathrm{hz}$. These waves denote relaxed state.[6]

4. Mu waves-Ranges between 8-12 Hz. Closely associated with motor activities.[6]

5. Beta waves-These waves lie between the range of 13$30 \mathrm{~Hz}$.Low beta waves is related to thinking and mental activity and high beta waves is related to tension, excitement, stress [6].

6. Gamma waves-They range between $30-42 \mathrm{~Hz}$.Associated with learning and memory information.[6]

7.

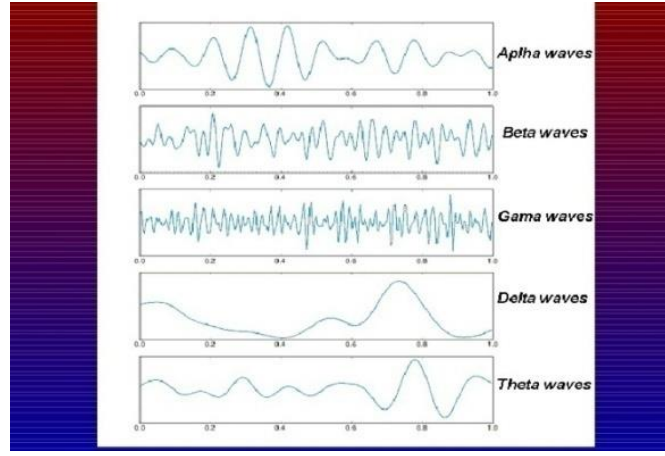

Figure 2: Different EEG brainwaves

\section{Algorithm}

\subsection{Valence arousal model}

The different emotions can be identified using the valence arousal values. It is a 2D model where the valence indicates the way one judges a situation and arousal indicates the excitement of a person [6]. Any emotion can be plotted using the valence arousal model.

The various emotions are grouped using Valence and arousal value. Based on the values the emotions are plotted on the quadrants.

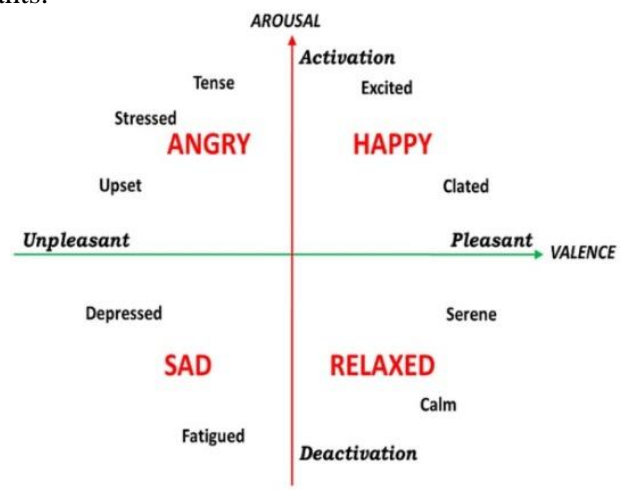

Figure 3: Valence arousal model

\subsection{Support vector machine (SVM)}

The Support Vector Machine is used to analyze the data for classification and regression. The SVM model is a illustration of points in space. They are divided into different categories and then mapped. In this algorithm each data item is a point in $\mathrm{n}$-dimensional space. The new points are then mapped into the same space and predicted to the category they belong to. SVM are frontiers that are used for separating the hyper-plane. To identify the correct hyper plane select the hyper plane that separates the two classes better.SVM are mainly used for the classification of images. Hand written characters can be identified using svm. Support vector machine has proved to be a prominent method in the past research works.

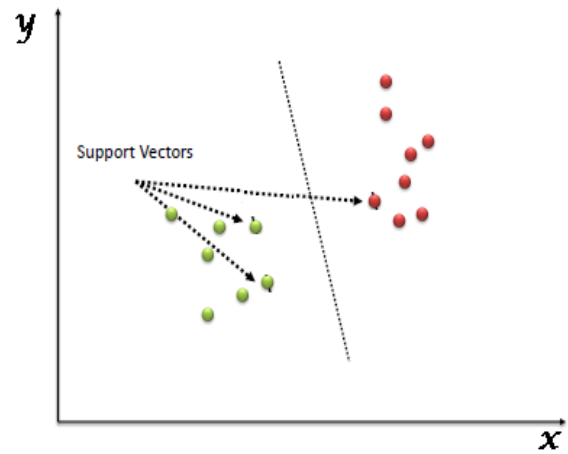

Figure 4: SVM 


\section{Data set collection}

For this proposed work, the data's has been collected from 64 subjects from various age groups. The majority of the data is collected from the teenagers because they are the ones who use social media for a long time compared to the other age groups. The data's have been recorded using the neurosky device. The workspace where we conducted the experiment was completely quiet and had no external disturbances. We ensure that the subjects feel really comfortable with the environment. The experimentation is divided into two partsThe first part is where the readings will be taken while using digital media and the other readings are taken after the meditation has been performed. Before meditation the subject is alerted that the process is starting and is asked to calm down. Then they are asked to focus on a particular object to achieve a calm state. Sudharshanakriya is a strong breathing technique that integrates natural rhythms of breath, harmonizing mind, body and emotions [4].This strategy eliminates the negative emotions like stress, fatigue, anger, depression and makes the mind calm and relaxed.

The experimental procedure involves the following steps-Data acquisition, Feature extraction, Data classification.

The initial step is to alert the subjects that the process is starting and asked them to calm down. The readings are taken before and after performing meditation. These questions were asked to the subjects before they sit for the test:

1. What is your age?

2. Gender: M F

3. What is your profession?

4. Any meditation experience? If so how many years?

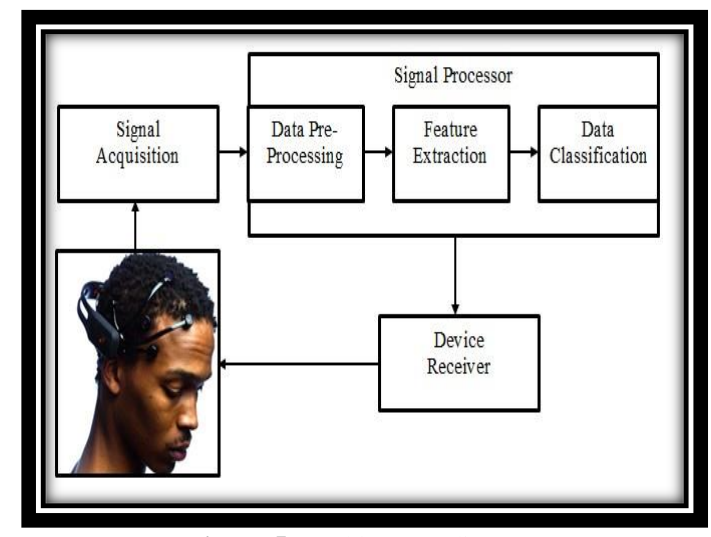

Figure 5: Architecture diagram

\section{Experimentation}

Firstly the subject is asked to sit in a quite environment. The device is fixed on the head and earclip position. The device is connected to a mobile via blue tooth. EEGid is a android application that is connected to the Neurosky Mindwave device.

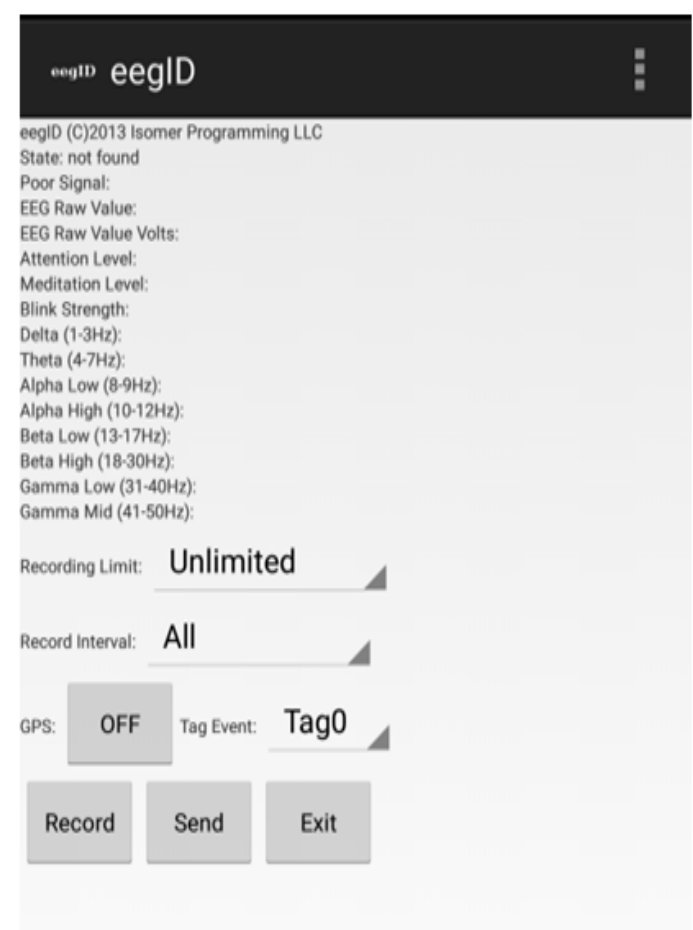

Figure 6: EEGid application

The time was set for 30 seconds and the readings were recorded. The first set of readings were taken when the subject is using digital media, and the next set of readings were taken after performing meditation. One of the advantages of neurosky device is that the unwanted signal or noise is removed. Neurosky consists of four sensors at the temporal and central lobes. This application records the readings taken from the device and is sent through email. The raw data is in CSV or excel format.

\section{Results}

The experiment was conducted to 64 subjects on various age groups, but majority of the readings were taken from the teenagers. The brain waves of each subject was recorded. The raw values were then normalised using the formula:

$$
=\frac{x-\min }{\max -\min }
$$




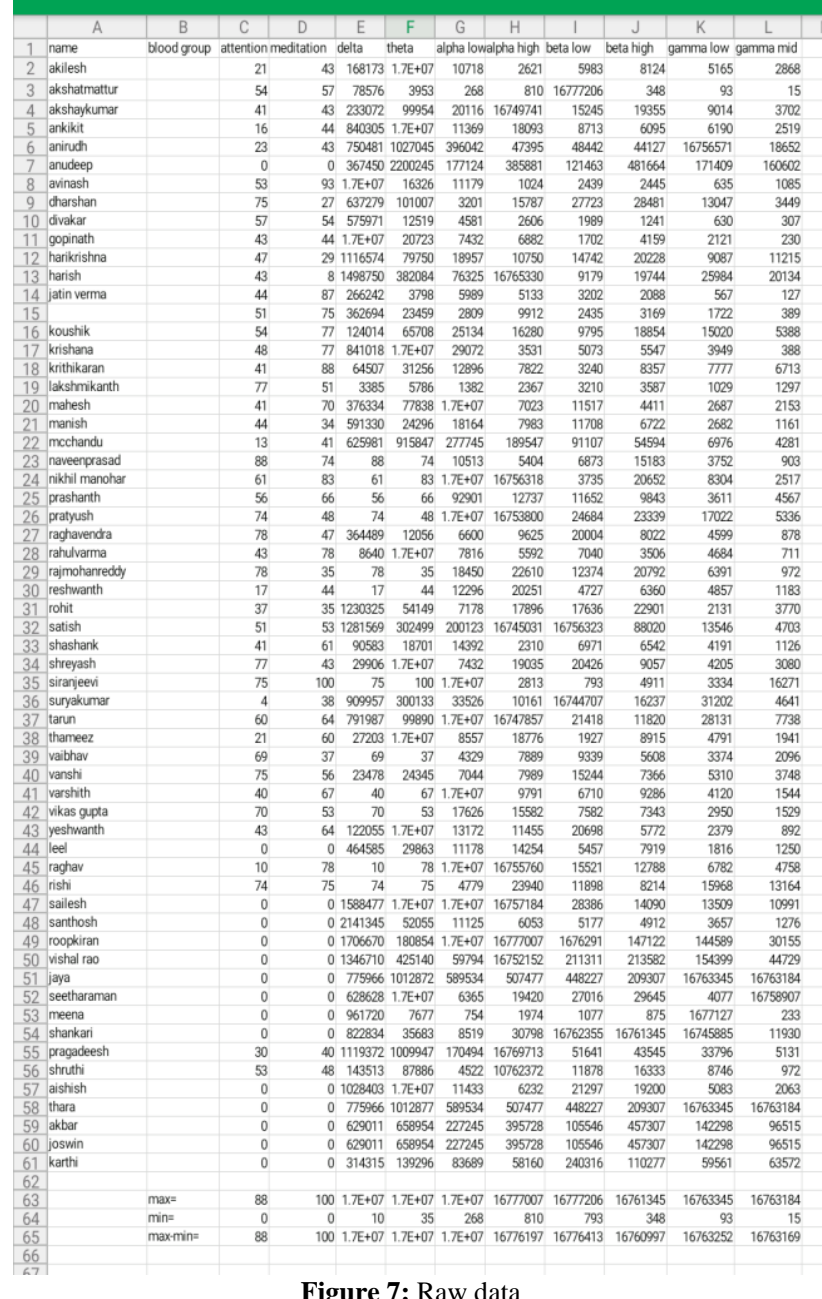

Figure 7: Raw data

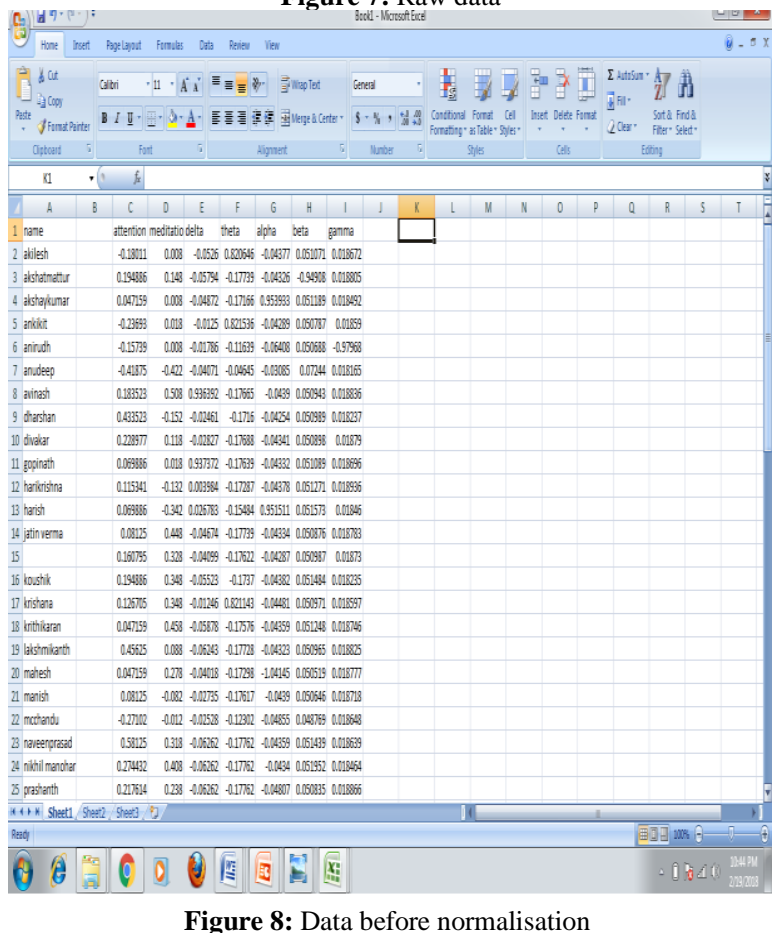

Figure 8: Data before normalisation

\begin{tabular}{|c|c|c|c|c|c|c|c|c|c|c|c|}
\hline A & $\begin{array}{lllllll}1 & 6 & 0 & 5 & 1 & 6 & H\end{array}$ & 11 & 1 & 1 & $\|$ & 1 & 0 & ? & a & \& & \\
\hline Iane & 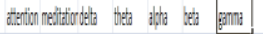 & & & & & & & & & & \\
\hline I bitent & 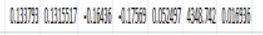 & & & & & & & & & & \\
\hline 3 destentalus & 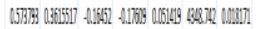 & & & & & & & & & & \\
\hline 4 astapunar & 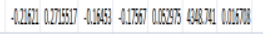 & & & & & & & & & & \\
\hline 5 alitit & 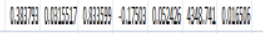 & & & & & & & & & & \\
\hline 6 airide & 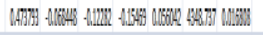 & & & & & & & & & & \\
\hline 7 nideet & 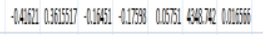 & & & & & & & & & & \\
\hline 8 aांको & 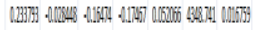 & & & & & & & & & & \\
\hline 9 dessiber & 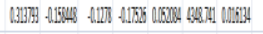 & & & & & & & & & & \\
\hline 111 bisige & 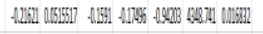 & & & & & & & & & & \\
\hline 11 pairibh & 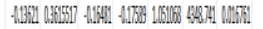 & & & & & & & & & & \\
\hline 12 havistita & 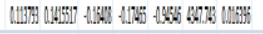 & & & & & & & & & & \\
\hline 13 histh & 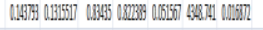 & & & & & & & & & & \\
\hline 19 intingma & 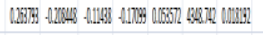 & & & & & & & & & & \\
\hline is & 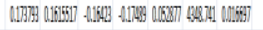 & & & & & & & & & & \\
\hline Ibagith & 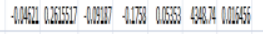 & & & & & & & & & & \\
\hline I1 histen & 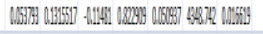 & & & & & & & & & & \\
\hline 13 hatilian & 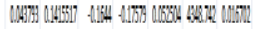 & & & & & & & & & & \\
\hline 13 istmilath & 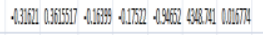 & & & & & & & & & & \\
\hline$y$ mateh & 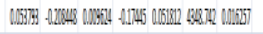 & & & & & & & & & & \\
\hline II mista & 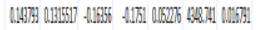 & & & & & & & & & & \\
\hline$n_{\text {mitanti }}$ & 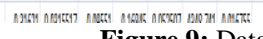 & & & & & & & & & & \\
\hline
\end{tabular}

Figure 9: Data after normalisation

After normalisation there is a variation in the alpha and beta waves. After meditation the subject is said to be in relaxed state.

The graph obtained from the data's is displayed below:

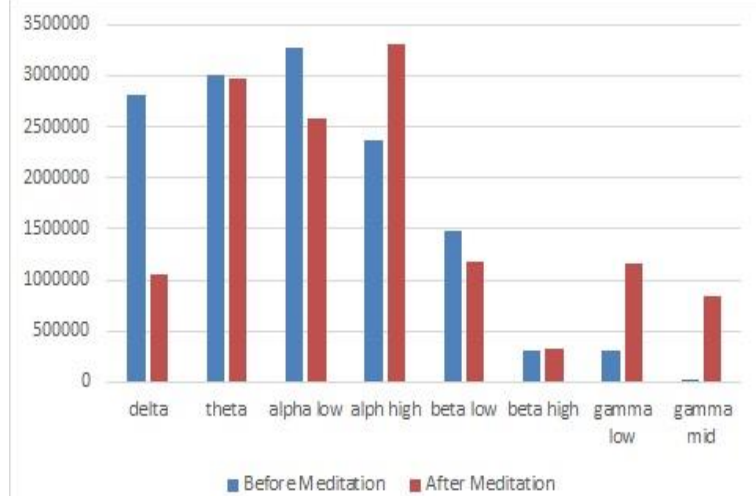

Figure 10: Variation in alpha and beta values before and after meditation

\section{Future enhancements}

This project is successful in reducing the negative emotions of the subjects. The subjects seem to be in a calm state after performing meditation. Hence using EEG a person's brain activity can be monitored and a solution can be provided. Individuals having stress and depression can perform meditation regularly to bring them to relaxed state.

\section{Conclusion}

The world is slowly transforming into the digital world. Though social media has lots of drawbacks we cannot stop using it, as it has become a necessary part of our life. Hence to overcome negative emotions, meditation is the best therapy. The obtained results shows that performing meditation regularly heals stress and depression. Altogether the subject seems to be in relaxed state after meditation. 


\section{References}

[1] Li J \& Oussalah M, "Automatic face emotion recognition system”, IEEE 9th International Conference on Cybernetic Intelligent Systems (CIS), (2010), pp.1-6.

[2] Koelstra S, Muhl C, Soleymani M, Lee JS, Yazdani A, Ebrahimi T \& Patras, I, "Deap: A database for emotion analysis; using physiological signals", IEEE Transactions on Affective Computing, Vol. 3, No. 1, (2012), pp.18-31.

[3] Surangsrirat D \& Intarapanich A, "Analysis of the meditation brainwave from consumer EEG device", IEEE Southeast Con, (2015), pp. 1-6.

[4] Kochupillai V, "Quantitative analysis of EEG signal before and after Sudharshana Kriya Yoga", Int J Public Mental Health and Neurosciences, Vol. 2, (2015), pp.19-22.

[5] Serrano-Puche J, "Emotions and digital technologies", Mapping the field of research in media studies, (2015).

[6] Uma M \& Sridhar SS, "A feasibility study for developing an emotional control system through brain computer interface", IEEE International Conference on Human Computer Interactions (ICHCI), (2013), pp. 1-6.

[7] Hygiea Casiano MD, Jolene Kinley MA, Laurence YKMD, Mariette JCRN \& Jitender SMD, "Media use and health outcomes in adoloscents: finding from a nationally representative survey", $J$ Can Acad Child Adolesc Psychiatry, Vol. 21, No. 4, (2012), pp. 296-301.

[8] Pea R, Nass C, Meheula L, Rance M, Kumar A, Bamford H \& Zhou M, "Media use, face-to-face communication, media multitasking, and social well-being among 8-to 12-year-old girls", Developmental Psychology, Vol. 48, No. 2, (2012), pp. 327-336.

[9] Thau M. Consciousness and cognition. Oxford University Press, (2002).

[10] Katona J, Farkas I, Ujbanyi T, Dukan P \& Kovari A, "Evaluation of the NeuroSky MindFlex EEG headset brain waves data", IEEE 12th International Symposium on Applied Machine Intelligence and Informatics (SAMI), (2014), pp.91-94.

[11] Kim J, Lee S, Kim S \& Yoo WY, "Music mood classification model based on arousal-valence values", IEEE 13th International Conference on Advanced Communication Technology (ICACT), (2011), pp.292-295. 